\title{
Artesunate Protects Against the Organ Injury and Dysfunction Induced by Severe Hemorrhage and Resuscitation
}

\author{
Regina Sordi, PhD, ${ }^{*}$ Kiran K. Nandra, PhD, ${ }^{*}$ Fausto Chiazza, BSc, $\dagger$ Florence L. Johnson, BSc, ${ }^{*}$ \\ Claudia P. Cabrera, PhD, $\ddagger$ Hew D. Torrance, MD, $\S$ Noriaki Yamada, MD, PhD, ${ }^{*}$ Nimesh S. A. Patel, PhD, * \\ Michael R. Barnes, PhD, $\ddagger$ Karim Brohi, MD, $\S$ Massimo Collino, PhD, $\dagger$ and Christoph Thiemermann, MD, PhD*
}

\begin{abstract}
Objective: To evaluate the effects of artesunate on organ injury and dysfunction associated with hemorrhagic shock (HS) in the rat.

Background: HS is still a common cause of death in severely injured patients and is characterized by impairment of organ perfusion, systemic inflammatory response, and multiple organ failure. There is no specific therapy that reduces organ injury/dysfunction. Artesunate exhibits pharmacological actions beyond its antimalarial activity, such as anticancer, antiviral, and anti-inflammatory effects.

Methods: Rats were submitted to HS. Mean arterial pressure was reduced to $30 \mathrm{~mm} \mathrm{Hg}$ for 90 minutes, followed by resuscitation. Rats were randomly treated with artesunate $(2.4 \mathrm{or} 4.8 \mathrm{mg} / \mathrm{kg}$ i.v.) or vehicle upon resuscitation. Four hours later, parameters of organ injury and dysfunction were assessed. Results: Artesunate attenuated the multiple organ injury and dysfunction caused by HS. Pathway analysis of RNA sequencing provided good evidence to support an effect of artesunate on the Akt-survival pathway, leading to downregulation of interleukin-1 receptor-associated kinase 1. Using Western
\end{abstract}

From the ${ }^{*}$ Centre for Translational Medicine and Therapeutics, Queen Mary University of London, William Harvey Research Institute, Barts and The London School of Medicine \& Dentistry, London, UK; †Department of Drug Science and Technology, University of Turin, Turin, Italy; $\ddagger$ Department of Clinical Pharmacology, Queen Mary University of London, William Harvey Research Institute, Barts and The London School of Medicine \& Dentistry, London, UK; and §Centre for Trauma Sciences, Queen Mary University of London, Blizard Institute, Barts and The London School of Medicine \& Dentistry, London, UK.

RS and KKN contributed equally to this work.

Disclosure: CT is inventor on a patent relating to the use of artesunate in hemorrhagic shock (United States Application No: 14/125032). RS is supported by the Program Science without Borders, CAPES Foundation, Ministry of Education of Brazil, Brasilia, DF, Brazil; KKN is supported by British Heart Foundation (grant no. FS/10/57/28485); NSAP is, in part, supported by the Bart's and The London Charity (753/1722). The research leading to these results has received funding from the People Programme (Marie Curie Actions) of the European Union's Seventh Framework Programme (FP7/ 2007-2013) under REA grant agreement no 608765, from the William Harvey Research Foundation and the University of Turin (Ricerca Locale ex-60\%). This work contributes to the Organ Protection research theme of the Barts Centre for Trauma Sciences, supported by the Barts and The London Charity (Award 753/1722) and forms part of the research themes contributing to the translational research portfolio of Barts and the London Cardiovascular Biomedical Research Unit that is supported and funded by the National Institute of Health Research. The funders had no role in study design, data collection and analysis, decision to publish, or preparation of the article.

The authors report no conflicts of interest.

Supplemental digital content is available for this article. Direct URL citations appear in the printed text and are provided in the HTML and PDF versions of this article on the journal's Web site (www.annalsofsurgery.com).

This is an open-access article distributed under the terms of the Creative Commons Attribution-Non Commercial-No Derivatives License 4.0, where it is permissible to download and share the work provided it is properly cited. The work cannot be changed in any way or used commercially.

Reprints: Professor Christoph Thiemermann, MD, PhD, FBPhS, FRCP, FMedSci, William Harvey Research Institute, Barts and The London School of Medicine \& Dentistry, Queen Mary University of London, Charterhouse Square, London EC1 M 6BQ, UK. E-mail: c.thiemermann@qmul.ac.uk.

Copyright (C) 2016 Wolters Kluwer Health, Inc. All rights reserved.

ISSN: 0003-4932/16/26502-0408

DOI: $10.1097 /$ SLA.0000000000001664 blot analysis, we confirmed that treatment of HS rats with artesunate enhanced the phosphorylation (activation) of Protein kinase B (Akt) and endothelial nitric oxide synthase and the phosphorylation (inhibition) of

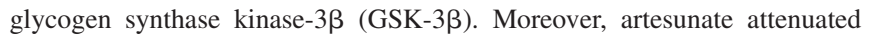
the HS-induced activation of nuclear factor kappa B and reduced the expression of proinflammatory proteins (inducible nitric oxide synthase, tumor necrosis factor- $\alpha$, and interleukin 6).

Conclusions: Artesunate attenuated the organ injury/dysfunction associated with HS by a mechanism that involves the activation of the Akt-endothelial nitric oxide synthase survival pathway, and the inhibition of glycogen synthase kinase-3 $\beta$ and nuclear factor kappa B. A phase II clinical trial evaluating the effects of good manufacturing practice-artesunate in patients with trauma and severe hemorrhage is planned.

Keywords: Akt, antimalarial, artesunate, hemorrhagic shock, interleukin-1 receptor-associated kinase 1 , nuclear factor kappa $\mathrm{B}$, organ injury

(Ann Surg 2017;265:408-417)

T rauma is a leading cause of death with 5 million of victims yearly. About $40 \%$ of trauma deaths are due to hemorrhagic shock (HS), which causes hypoperfusion of organs leading to multiple organ failure (MOF). ${ }^{1}$ MOF occurs in $30 \%$ of injured patients, and it is the major cause of morbidity after trauma. ${ }^{2}$ MOF correlates with an increase in nosocomial infections and, if it persists, mortality. ${ }^{3}$ The mechanisms underlying MOF are not fully understood, but ischemiareperfusion injury and systemic inflammation are key players. ${ }^{4}$ Although there are clear guidelines for the management of HS and organ support strategies, there is no specific pharmacological intervention that prevents the MOF associated with HS. Thus, a therapeutic agent that reduces the incidence and/or severity of MOF is urgently needed and could have major global impact on patient outcomes.

Artesunate (a semisynthetic artemisinin derived from Artemisia annua) is recommended by the World Health Organization as the treatment of choice for severe malaria. ${ }^{5}$ Artesunate is a safe, lowcost drug, which has been used by thousands of patients with malaria without important adverse effects. ${ }^{6}$ Although the mechanism behind the antimalarial activity of artesunate is not entirely clear, it is thought that the active metabolite, dihydroartemisinin is responsible for the death of the parasite. ${ }^{7}$

Artemisinins, including artesunate, have many pharmacological effects beyond the eradication of Plasmodium. ${ }^{8}$ Artemisinins/ artesunate are cytotoxic against cancer cells,,${ }^{9}$ viruses,,${ }^{10}$ fungi,,${ }^{11}$ but also exert powerful anti-inflammatory effects in experimental asthma, ${ }^{12}$ pancreatitis, ${ }^{13}$ arthritis,${ }^{14}$ and sepsis ${ }^{15}$. The effects of artesunate in HS are unknown. Therefore, the aim of the present study was to investigate the effects of artesunate on the organ injury/ dysfunction associated with HS. Having discovered that artesunate attenuates the MOF associated with HS, we have investigated the potential mechanism of action of artesunate, including RNA-seq transcriptomics and pathway analysis. We report here for the first time that artesunate has prosurvival and anti-inflammatory 
properties, as it activates the Akt-endothelial nitric oxide synthase (eNOS) cell survival pathway and inhibits a range of proinflammatory molecules, including interleukin-1 receptor-associated kinase 1 (IRAK1), forkhead box O4 (FOXO4), glycogen synthase kinase-3 $\beta$ $(\mathrm{GSK}-3 \beta)$, and the transcription factor nuclear factor kappa B (NF-кB).

\section{METHODS}

\section{Animal Welfare and Ethical Statements}

The animal protocols used in this study were approved by the Animal Welfare Ethics Review Board of Queen Mary University of London (PPL: 70/7348) in accordance with Home Office guidance on Operation of Animals (Scientific Procedures Act 1986) and Guide for Care and Use of Laboratory Animals of the National Research Council.

\section{Hemorrhagic Shock and Quantification of Organ Injury}

This study was carried out on 120 male Wistar rats (Charles River Ltd, UK) weighing $\sim 280 \mathrm{~g}$ receiving a standard diet and water ad libitum. HS was performed as previously described. ${ }^{16}$ Rats were anesthetized using sodium thiopentone $(120 \mathrm{mg} / \mathrm{kg}$ i.p. maintained using $10 \mathrm{mg} / \mathrm{kg}$ i.v.). Blood was withdrawn to achieve a fall in mean arterial pressure (MAP) to $30 \pm 2 \mathrm{~mm} \mathrm{Hg}$, which was maintained for a period of 90 minutes. At 90 minutes after initiation of hemorrhage (or when $25 \%$ of the shed blood had to be reinjected to sustain MAP at $30 \mathrm{~mm} \mathrm{Hg}$ ), resuscitation was performed with the (remaining) shed blood over a period of 5 minutes. The same volume of blood reinfused for maintenance of MAP was replaced by Ringer lactate on resuscitation. An infusion of Ringer Lactate ( $1.5 \mathrm{~mL} / \mathrm{kg} / \mathrm{hour}$; i.v.) was maintained throughout the experiment for a total of 4 hours. The last 3 hours urine was obtained for the estimation of creatinine clearance. Blood was collected for the measurement of lactate (Accutrend Plus Meter, Roche Diagnostics, UK) and organ injury parameters (IDEXX Ltd, UK). Samples were taken and stored at $-80^{\circ} \mathrm{C}$. Sham rats were used as control and underwent identical surgical procedures, but without hemorrhage/resuscitation.

\section{Experimental Design}

Rats were randomly allocated into the following groups $(\mathrm{n}=$ 10 per group): sham + vehicle; sham + artesunate $4.8 \mathrm{mg} / \mathrm{kg}$; HS + vehicle; $\mathrm{HS}+$ artesunate $2.4 \mathrm{mg} / \mathrm{kg}$ and $\mathrm{HS}+$ artesunate $4.8 \mathrm{mg} / \mathrm{kg}$. Artesunate was diluted in phosphate buffer (vehicle; $\mathrm{NaH}_{2} \mathrm{PO}_{4}$ $18.3 \mathrm{mM} ; \mathrm{Na}_{2} \mathrm{HPO}_{4} 150.4 \mathrm{mM}$; $\mathrm{pH}$ 7.9-8.1). Rats were administered vehicle $(1 \mathrm{~mL} / \mathrm{kg})$ or artesunate $(2.4 \mathrm{mg} / \mathrm{mL} / \mathrm{kg}$ or $4.8 \mathrm{mg} / \mathrm{mL} / \mathrm{kg})$ i.v. upon resuscitation. We have performed a previous study designed to evaluate the effects of artesunate $(1,3$, and $10 \mathrm{mg} / \mathrm{kg}$; supplemental Fig. 1, http://links.lww.com/SLA/A977) in HS. The maximal reduction in organ injury/dysfunction was reached with a dose of $3 \mathrm{mg} / \mathrm{kg}$. To increase the clinical relevance (and potentially the ability to translate our preclinical findings to a clinical trial), we have then evaluated the effects of $2.4 \mathrm{mg} / \mathrm{kg}$ of artesunate in HS, as this dose is given to patients with severe malaria. We have also investigated a higher dose of artesunate $(4.8 \mathrm{mg} / \mathrm{kg})$.

\section{Determination of Myeloperoxidase Activity} described. ${ }^{16}$

Myeloperoxidase (MPO) activity was performed as previously

\section{RNA sequencing Analysis}

Gene expression was evaluated by RNA sequencing (RNA-seq) that allows a dynamic range of detection and measurement of relatively limited differences in expression between samples. The transcriptomic analysis included a normalization procedure that should correct for differential RNA yields between samples. Whole blood RNA samples were extracted from 12 rats ( 3 samples per group). One microgram of total RNA was used for library preparation, according to the Illumina TruSeq mRNA sample preparation protocol (Illumina, San Diego, CA). Libraries were sequenced using Illumina NextSeq platform and TruSeq protocol. Adaptor and poor quality sequences were removed using trim galore (v 0.3.7) software, Babraham, Cambridgeshire, UK. Trimmed sequences were aligned to the rat reference genome (rnor5) with TopHat2 (v2.0.13)/bowtie2 (v2.2.3), Baltimore, MD. Read counts were calculated using the HTSeq-counts program and differential expression analyses were carried out with DESeq2, using the standard protocols. Pathway analysis was performed using Enrichr ${ }^{17}$ and Ingenuity Pathway Analysis.

\section{Western Blot}

Western blot was performed as previously described. ${ }^{16}$ The densitometric analysis was performed using Gel Pro Analyzer 4.5, 2000 software (Media Cybernetics, Silver Spring, MD) and is expressed as relative optical density, corrected for the corresponding $\beta$-actin or histone $\mathrm{H} 3$ contents, and normalized using the related mean sham-operated band.

\section{Immunostaining for 3-Nitrotyrosine}

Immunostaining was performed using anti-3-nitrotyrosine (3NT) antibody (1:2000; Merck Millipore, Germany) and the kit Dako EnVision + System-HRP-DAB. The quantification of immunostaining was achieved through densitometry analysis of 10 random fields of each sample using NIH ImageJ 1.36 imaging software (NIH, Bethesda, MD).

\section{Cytokines}

Serum cytokines tumor necrosis factor (TNF)- $\alpha$ and interleukin (IL)-6 were determined using commercial immunoassay kits (R\&D Systems, Minneapolis, MN) according to the manufacturer protocol.

\section{Materials}

Unless otherwise stated, all compounds were from SigmaAldrich Company Ltd (Poole, Dorset, UK). Ringer's Lactate was from Baxter Healthcare Ltd (Deerfield, IL); sodium thiopentone (Thiovet) from Link Pharmaceuticals, Horsham, UK. The bicinchoninic acid protein assay kit and SuperBlock blocking buffer were from Thermo Fisher Scientific Inc (Rockford, IL). Antibodies were from Cell Signalling Technology Inc (Beverly, MA).

\section{Data Analysis}

All figures are expressed as box-and-whisker format showing medians, interquartile range, and full range. The tables are showing minimun, $25 \%$ percentile, median, $75 \%$ percentile and maximum values. Statistical analysis was carried out using GraphPad Prism 5.03 (GraphPad Software, La Jolla, CA). Data were assessed by 1 or 2-way analysis of variance followed by Bonferroni test. The distribution of the data was verified by Kolmogorov-Smirnov test, and the homogeneity of variances by Bartlett test. When necessary, values have been transformed into logarithmic values to achieve normality and homogeneity of variances. The data that were not normally distributed were analyzed with a nonparametric test (Kruskal-Wallis followed by Dunn test). A P $<0.05$ was considered to be significant.

\section{RESULTS}

\section{Artesunate Attenuates the Organ Injury and Dysfunction Induced by HS}

When compared to sham rats, rats subjected to HS exhibited a decrease in creatinine clearance (Fig. 1A) and an increase in serum creatinine (Fig. 1B) and, hence, renal dysfunction. HS rats also 

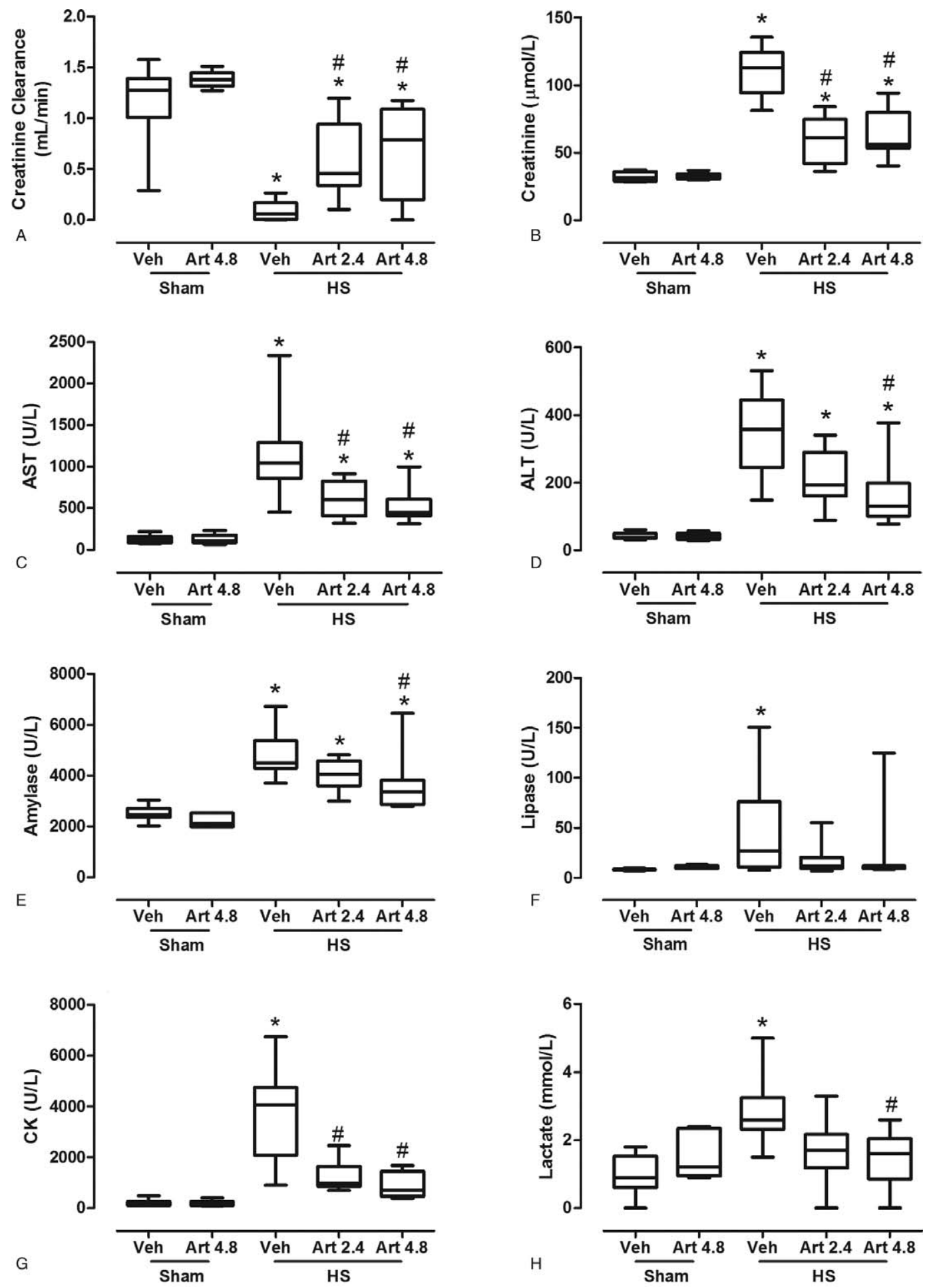

FIGURE 1. Artesunate attenuates the organ injury and dysfunction induced by HS. A, creatinine clearance; B, serum creatinine; $C$, serum AST; D, serum alanine ALT; E; serum amylase; F, serum lipase; $G$, serum CK; and $H$, lactate of HS rats treated with vehicle or artesunate $(2.4$ or $4.8 \mathrm{mg} / \mathrm{kg}$ ) on resuscitation are shown. Sham rats were treated with vehicle or artesunate $4.8 \mathrm{mg} / \mathrm{kg}$. Data are presented as box and whiskers, showing medians, interquartile range, and full range ( $\mathrm{n}=10$ animals per group). When necessary (as lipase data), the values had been transformed into logarithms to achieve normality and homogeneity of variances. Statistical analysis was performed using 1-way ANOVA followed by Bonferroni post hoc test. ${ }^{*} P<0.05$ vs sham + vehicle and ${ }^{\#} P<0.05$ vs $\mathrm{HS}+$ vehicle. ANOVA indicates analysis of variance; CK, creatine kinase. 
developed significant increases in serum aspartate aminotransferase (Fig. 1C), serum alanine aminotransferase (Fig. 1D), serum amylase (Fig. 1E), serum lipase (Fig. 1F), serum creatine kinase (Fig. 1G), and lactate $($ Fig. $1 \mathrm{H})$ indicating the development of liver, pancreas, and neuromuscular injury, and global ischemia, respectively (Fig. 1).

When compared to HS rats treated with vehicle, treatment of HS rats with $2.4 \mathrm{mg} / \mathrm{kg}$ or $4.8 \mathrm{mg} / \mathrm{kg}$ artesunate attenuated the fall in creatinine clearance (Fig. 1A) and attenuated the rises in serum creatinine (Fig. 1B), aspartate aminotransferase (Fig. 1C), and creatine kinase (Fig. 1G). Treatment of HS rats with $4.8 \mathrm{mg} / \mathrm{kg}$ artesunate also significantly attenuated the HS-induced rise in serum levels of alanine aminotransferase (Fig. 1D), amylase (Fig. 1E), and lactate (Fig. 1H). No statistical difference was found between any of the groups studied in serum glucose levels (supplemental Table 1, http://links.lww.com/SLA/A977).

When compared to sham-operated rats, HS rats developed a significant increase in lung MPO activity (Fig. 2) indicating lung infiltration with inflammatory cells. Treatment of HS rats with both doses of artesunate significantly attenuated the rise in MPO activity in the lung (Fig. 2). Administration of high-dose artesunate to shamoperated rats had no significant effect on any of the parameters evaluated.

\section{Artesunate Does Not Prevent the Development of the Circulatory Failure Induced by HS}

When compared to sham-operated rats (open circles), HS rats treated with vehicle demonstrated a significant reduction in MAP after resuscitation $(P<0.05$; closed squares; Fig. 3). Administration of artesunate $(2.4$ or $4.8 \mathrm{mg} / \mathrm{kg}$; closed triangles) on resuscitation failed to attenuate the reduction in MAP caused by HS (Fig. 3) and had no effect on MAP of sham rats (closed diamonds). Heart rate (BPM) measurements of all groups of animals are shown in supplemental Table 2, http://links.lww.com/SLA/A977.

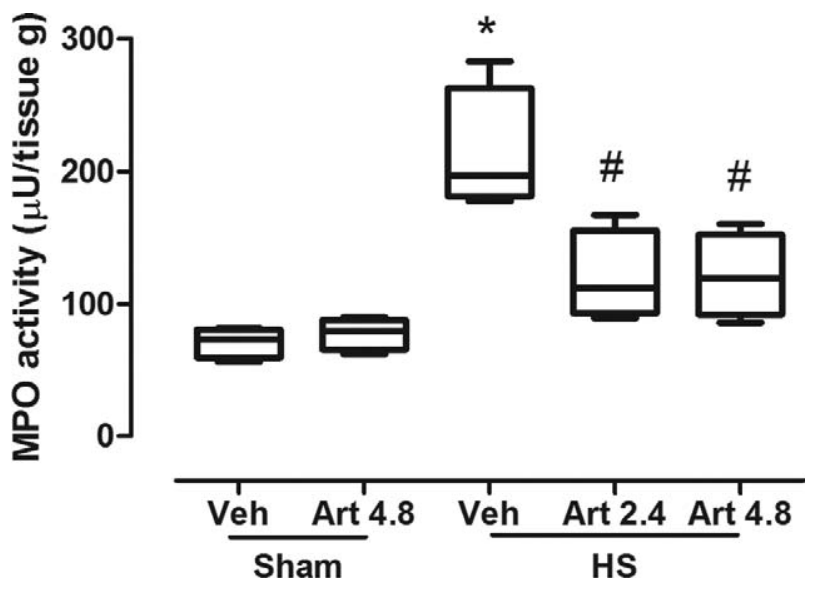

FIGURE 2. Artesunate attenuates MPO activity in lungs induced by HS. MPO activity in lungs of sham and HS rats treated with vehicle or artesunate $(2.4 \mathrm{or} 4.8 \mathrm{mg} / \mathrm{kg}$ ) on resuscitation are shown. Sham rats were treated with vehicle or artesunate $4.8 \mathrm{mg} / \mathrm{kg}$. Data are presented as box and whiskers, showing medians, interquartile range, and full range $(n=5$ animals per group). Statistical analysis was performed using 1 way ANOVA followed by Bonferroni post hoc test. ${ }^{*} P<0.05$ vs sham + vehicle and ${ }^{\#} P<0.05$ vs $\mathrm{HS}+$ vehicle. ANOVA indicates analysis of variance.

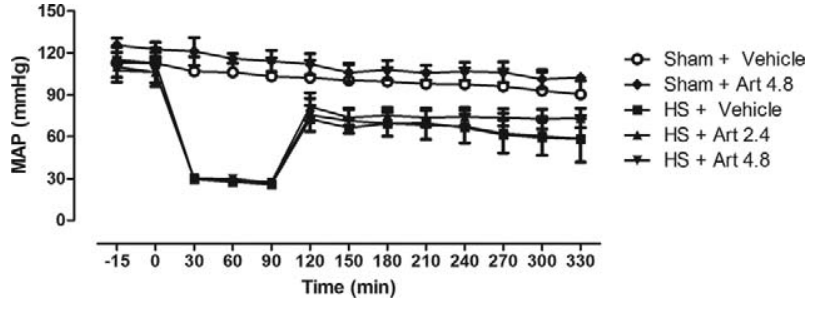

FIGURE 3. Artesunate does not prevent the development of the circulatory failure induced by HS. HS rats were treated with vehicle or artesunate $(2.4$ or $4.8 \mathrm{mg} / \mathrm{kg})$ on resuscitation. Sham animals were used as control and received vehicle or artesunate $(4.8 \mathrm{mg} / \mathrm{kg})$. The MAP was recorded during the whole experiment. Data are expressed as mean $\pm S D$ of 10 animals per group. Statistical analysis was performed using 2-way ANOVA followed by Bonferroni post hoc test. $P<0.05$ sham vehicle vs $\mathrm{HS}+$ vehicle (not indicated in the figure). ANOVA indicates analysis of variance; SD, standard deviation.

\section{Artesunate Acts Predominantly on the AKT-survival Pathway \\ RNA-seq transcriptome sequencing was performed on white} blood cells. In order to evaluate the key molecular drivers of the observed differential expression in both sham and HS rats, we evaluated enrichment of known protein-protein interaction hubs using the Enrichr analysis tool (supplemental Fig. 2, http://links.lww.com/SLA/A977). This identified, AKT1 protein interaction as the consistently strongest protein-protein interaction enrichment on a genome wide basis in both comparisons (10/119 AKT1 target genes show differential expression; adjusted $P=0.00116$; supplemental Fig. 2, http://links.lww.com/SLA/A977, and supplemental Table 3, http://links.lww.com/SLA/A977). The AKT1 interactor genes identified in the analysis were PPM1A, IRAK1, AKT2, ITGB3, LMNA, NCOA4, BPGM, FOXO4, BCL2L1, and CDC25B. To investigate the possible mechanistic basis of AKT1 interaction further, we constructed a mechanistic model of differentially expressed direct AKT1 interactors using the molecule activity predictor algorithm in Ingenuity Pathway Analysis. ${ }^{18}$ The algorithm predicted AKT activation (Fig. 4), on the basis of downregulation of a number of genes in the AKT core network that are known to be downregulated by AKT, including IL-1 receptor-associated kinase 1 and forkhead box $\mathrm{O} 4$.

\section{Artesunate Attenuates the Activation of NF-кB Induced by HS}

As artesunate may activate the Akt-survival pathway (Fig. 4), and as organ protection by artesunate were observed in liver/kidney (Fig. 1), we investigated the possible mechanisms by which artesunate $(4.8 \mathrm{mg} / \mathrm{kg})$ attenuated organ injury in liver/kidney. When compared to sham rats, we found no significant differences in the phosphorylation of Akt on $\mathrm{Ser}^{473}$ (Figs. 5A, 6A), eNOS on Ser ${ }^{1177}$ (Figs. 5B, 6B) or GSK-3 $\beta$ on $\mathrm{Ser}^{9}$ (Figs. 5C, 6C) in liver/kidney, from HS rats treated with vehicle. HS rats, however, exhibited an increase in the nuclear translocation of the p65 NF- $\mathrm{BB}$ subunit (Figs. 5D, 6D). Treatment of HS rats with artesunate resulted in a significant increase in the phosphorylation of Akt (Figs. 5A, 6A), eNOS (Figs. 5B, 6B), GSK-3 $\beta$ (Figs. 5C, 6C), and attenuated the translocation of the p65 NF-кB subunit to the nucleus (Figs. 5D, $6 \mathrm{D})$. The treatment of sham rats with artesunate had no significant effect (Figs. 5, 6). 


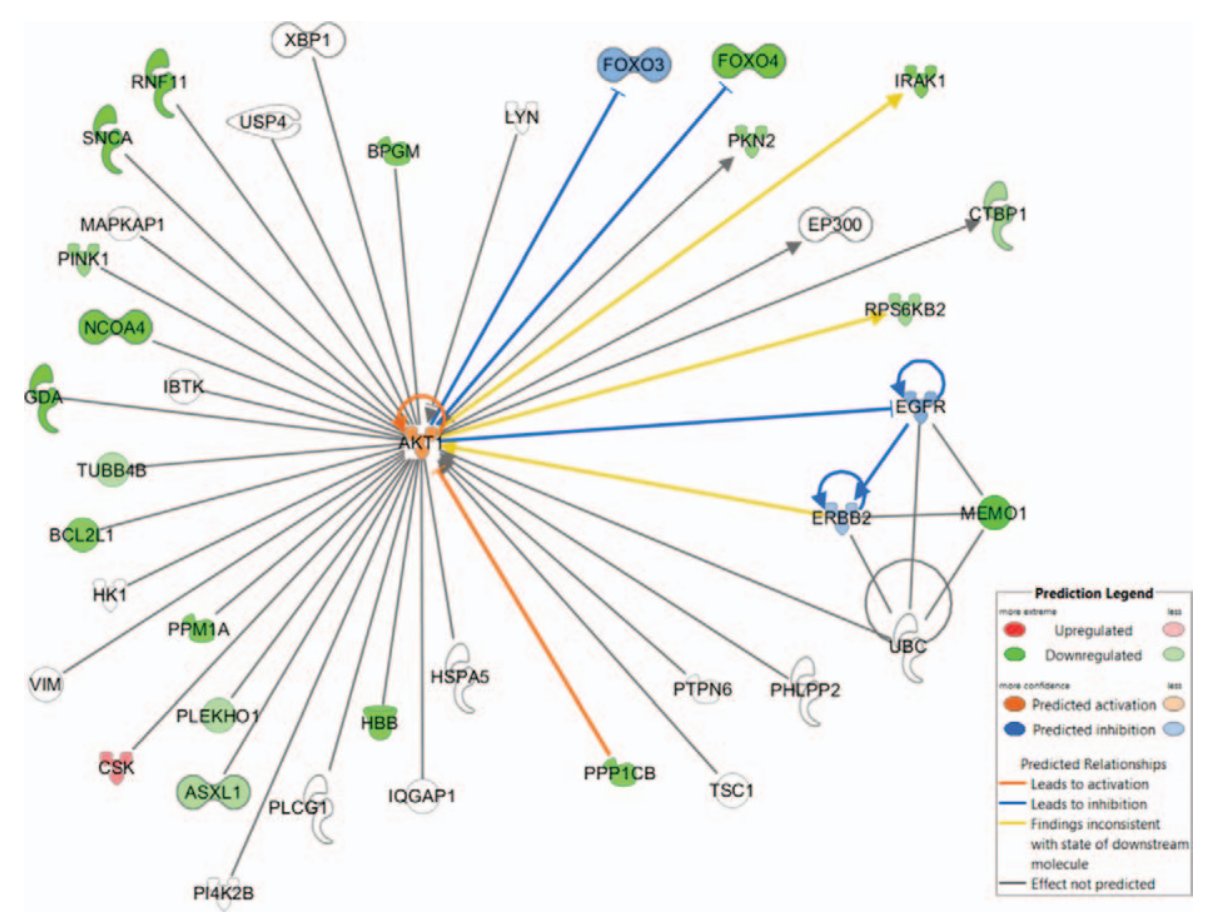

FIGURE 4. Core network of AKT1 interactor proteins constructed using Ingenuity pathway analysis. The network was constructed using AKT1 as a seed and all genes showing differential expression in $\mathrm{SH} \vee \mathrm{SH}+\mathrm{ART}$ and HS $\mathrm{H}$ HS + ART analysis. Ingenuity Molecule Activity Predictor analysis predicts activation of AKT1 on the basis of observed downregulation in several interactors that are known to be inhibited by AKT1. AKT1 indicates Core network of AKT1 gene interactor proteins; ART, artesunate; sham.

\section{Artesunate Reduces the iNOS Expression and Cytokine Formation Induced by HS}

As artesunate caused a decrease in the nuclear translocation of p65 NF- $\mathrm{B}$, we also investigated the effects of artesunate on the expression of NF- $\mathrm{KB}$-dependent proinflammatory proteins. When compared with sham-operated rats, livers and kidneys from rats submitted to HS exhibited a significant increase in iNOS expression (Figs. 7A, B, respectively). The treatment of HS rats with artesunate at the onset of resuscitation significantly attenuated the iNOS expression caused by HS in livers and kidneys (Figs. 7A, B). Although there was a trend for an increase in 3-NT staining in HS animals, we observed no significant difference in 3-NT staining in liver or kidney in any of the groups (supplemental Fig. 3, http:// links.lww.com/SLA/A977).

When compared to sham-operated rats, HS rats treated with vehicle developed significant increases in the serum levels of the proinflammatory cytokines TNF- $\alpha$ (Fig. 7C) and IL-6 (Fig. 7D). The treatment of HS rats with artesunate significantly attenuated the increases in serum TNF- $\alpha$ and IL- 6 caused by HS (Fig. 7C, D). The treatment of sham rats with artesunate had no effect on serum cytokine levels.

\section{DISCUSSION}

The main finding of the present report is that the antimalarial drug artesunate attenuates the organ injury and dysfunction associated with HS. Administration of artesunate upon resuscitation reduces the MOF by enhancing the resistance of organs against injury/dysfunction by activating a well-known cell survival pathway and by reducing excessive inflammation. The lower dose of artesunate shown here to be effective in HS is identical to the dose used in patients with malaria, many of which also have multiple organ dysfunction. Indeed, in these patients artesunate is superior to quinine in improving survival. ${ }^{6}$
In order to gain a better insight into the potential mechanism of action of artesunate in HS, we carried out a controlled RNA-seq transcriptome analysis of white blood cells. Pathway analysis of genes showing differential expression in both sham and HS rats was strongly enriched for AKT1 interactor genes. Although AKT1 itself was not differentially expressed, this does not reflect protein activity and a mechanistic model of the AKT1 core network-predicted AKT activation, on the basis of downregulation of several proinflammatory genes in the AKT core network that are known to be downregulated by AKT. These include IRAK1 and FOXO4. IRAK1 induces nuclear translocation of NF- $\mathrm{BB}$ and activator protein AP1 , resulting in the production of TNF- $\alpha$ and IL- $6 .{ }^{19}$ Downregulation of IRAK1 is protective in ischemia/reperfusion injury. ${ }^{20}$ FOXO4 specifically activates MMP9 expression in response to TNF- $\alpha$, acting as a convergence point of cytokine and growth factor signaling pathways. Thus, inhibition (or downregulation as seen with artesunate) of FOXO4 could provide a therapeutic target for inflammatory arterial diseases. $^{21}$

In our gene expression studies, we also observed a decrease in gene expression of MEMO1 in sham-operated animals and in HS rats (data not shown). This finding was somewhat contradictory to the hypothesis of AKT activation, as the protein has been shown to activate the Akt pathway. ${ }^{22}$ Subsequently, we have evaluated MEMO1 by Western blot analysis and found a consistent increase in MEMO1 protein, confirming that artesunate does indeed enhance the expression of MEMO1 protein in liver/kidney of sham and HS rats (data not shown). The observed reduction in MEMO1 transcript expression may represent a feedback control mechanism.

We discovered that treatment of HS rats with artesunate activates the kinase $\mathrm{Akt},{ }^{23}$ activation of which has beneficial effects including reduction/prevention of i) ventilation-induced lung injury, ${ }^{24}$ ii) sepsis-induced cardiac dysfunction, ${ }^{25}$ iii) sepsis-induced acute kidney injury, ${ }^{26}$ iv) myocardial ischaemia-reperfusion, ${ }^{27}$ and v) HS-induced organ dysfunction. ${ }^{28}$ Activation of Akt results in 

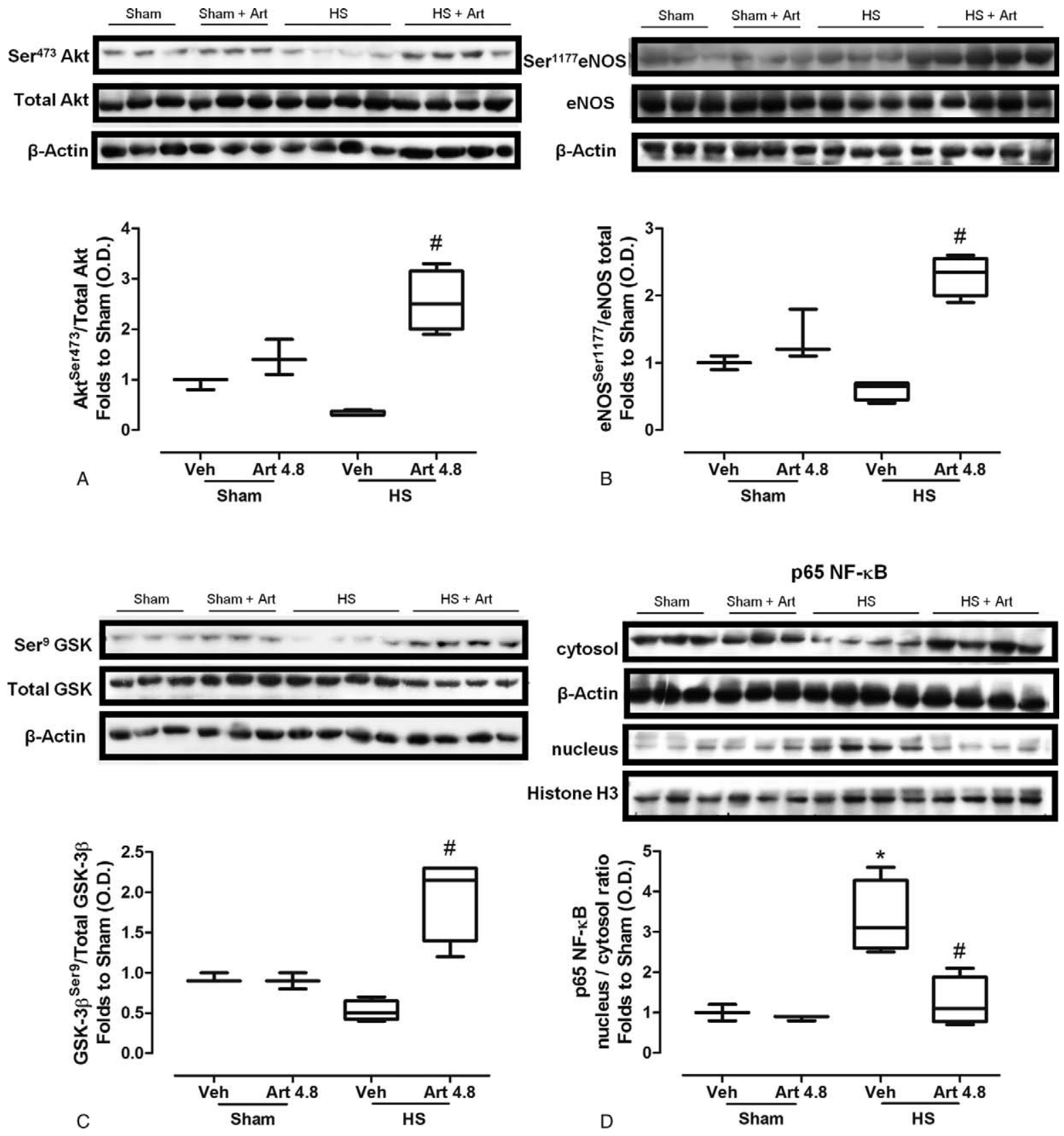

FIGURE 5. Artesunate attenuates the activation of NF-кB pathway induced by HS in the liver. The phosphorylation of (A) Ser ${ }^{473}$ on

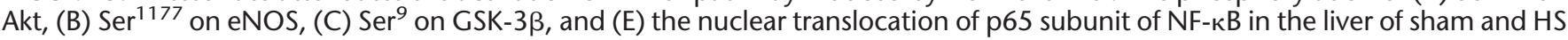
rats treated with vehicle or artesunate $(4.8 \mathrm{mg} / \mathrm{kg})$ on resuscitation were determined by Western blotting. Protein expression was measured as relative OD, corrected for the corresponding $\beta$-actin or histone contents and normalized using the related sham band. Data are presented as box and whiskers, showing medians, interquartile range, and full range ( $n=3-4$ animals per group). Statistical analysis was performed using Kruskal-Wallis test followed by Dunn post hoc test. ${ }^{*} P<0.05$ vs sham + vehicle and ${ }^{\#} P<0.05$ vs HS + vehicle. OD indicates optical density.

phosphorylation and activation of eNOS at Ser $1177^{29}$ that enhances the formation of small amounts of $\mathrm{NO}$, which is pivotal for the preservation of microvascular perfusion, ${ }^{30}$ reducing organ injury. ${ }^{25,31}$

Activation of Akt was also associated with an increase in phosphorylation of GSK-3 $\beta$. Phosphorylation of the constitutivelyactive, serine-threonine kinase GSK-3 $\beta$ by Akt turns off the catalytic activity of GSK-3 $\beta^{23}$. Inhibition of GSK-3 $\beta$ reduces inflammation ${ }^{32}$ and organ injury in myocardial ischemia, ${ }^{25}$ colitis, ${ }^{33}$ endotoxemia, ${ }^{34,35}$ and $\mathrm{HS}^{36}$ Thus, activation of Akt/eNOS and inactivation of GSK-3 $\beta$ may contribute to the beneficial effects of artesunate in $\mathrm{HS}$.

Inhibition of GSK-3 $\beta$ directly inhibits $\mathrm{NF}-\kappa \mathrm{B}$-dependent gene transcription, ${ }^{37}$ and NF- $\mathrm{KB}$ plays a pivotal role in inflammation ${ }^{38}$ and 

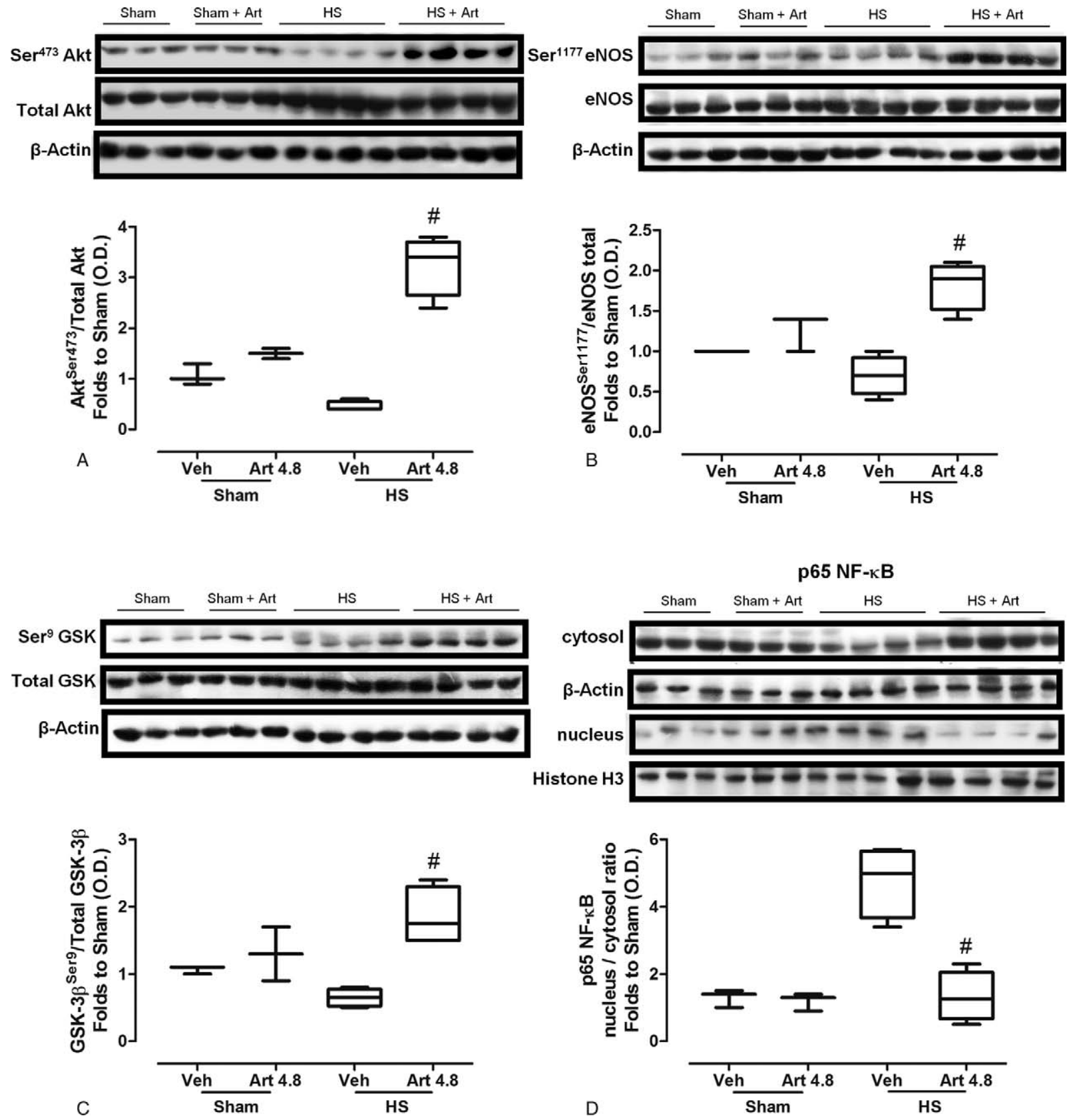

FIGURE 6. Artesunate attenuates the activation of NF-kB pathway induced by HS in the kidney. The phosphorylation of (A) Ser 473

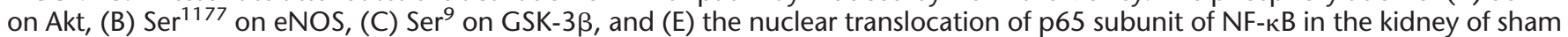
and HS rats treated with vehicle or artesunate $(4.8 \mathrm{mg} / \mathrm{kg})$ on resuscitation were determined by Western blotting. Protein expression was measured as relative optical density (OD), corrected for the corresponding $\beta$-actin or histone contents and normalized using the related sham band. Data are presented as box and whiskers, showing medians, interquartile range and full range $(n=3-4$ animals per group). Statistical analysis was performed using Kruskal-Wallis test followed by Dunn post hoc test. ${ }^{\#} P<0.05$ vs HS + vehicle. OD indicates optical density.

organ injury ${ }^{28,31,39}$. HS increased the translocation of subunit $\mathrm{p} 65$ to the nucleus, indicating NF- $\mathrm{B}$ activation. Treatment of HS rats with artesunate caused inactivation of GSK-3 $\beta$ (phosphorylation), which was associated with reduced nuclear translocation of p65. Indeed, inhibition of NF-кB (by inhibiting activation of the Inhibitor of карpa $\mathrm{B}$ kinase complex) reduces the organ injury/inflammation in HS. ${ }^{28}$
Thus, inhibition of GSK-3 $\beta$ and NF-кB may both importantly contribute to the observed anti-inflammatory effects of artesunate in HS.

As NF- $\kappa \mathrm{B}$ regulates iNOS expression and iNOS plays an important role in the pathophysiology of $\mathrm{HS},{ }^{40,41}$ we have investigated the effects of artesunate on iNOS expression in HS rats. HS increased the expression of iNOS in liver and kidney, and artesunate 

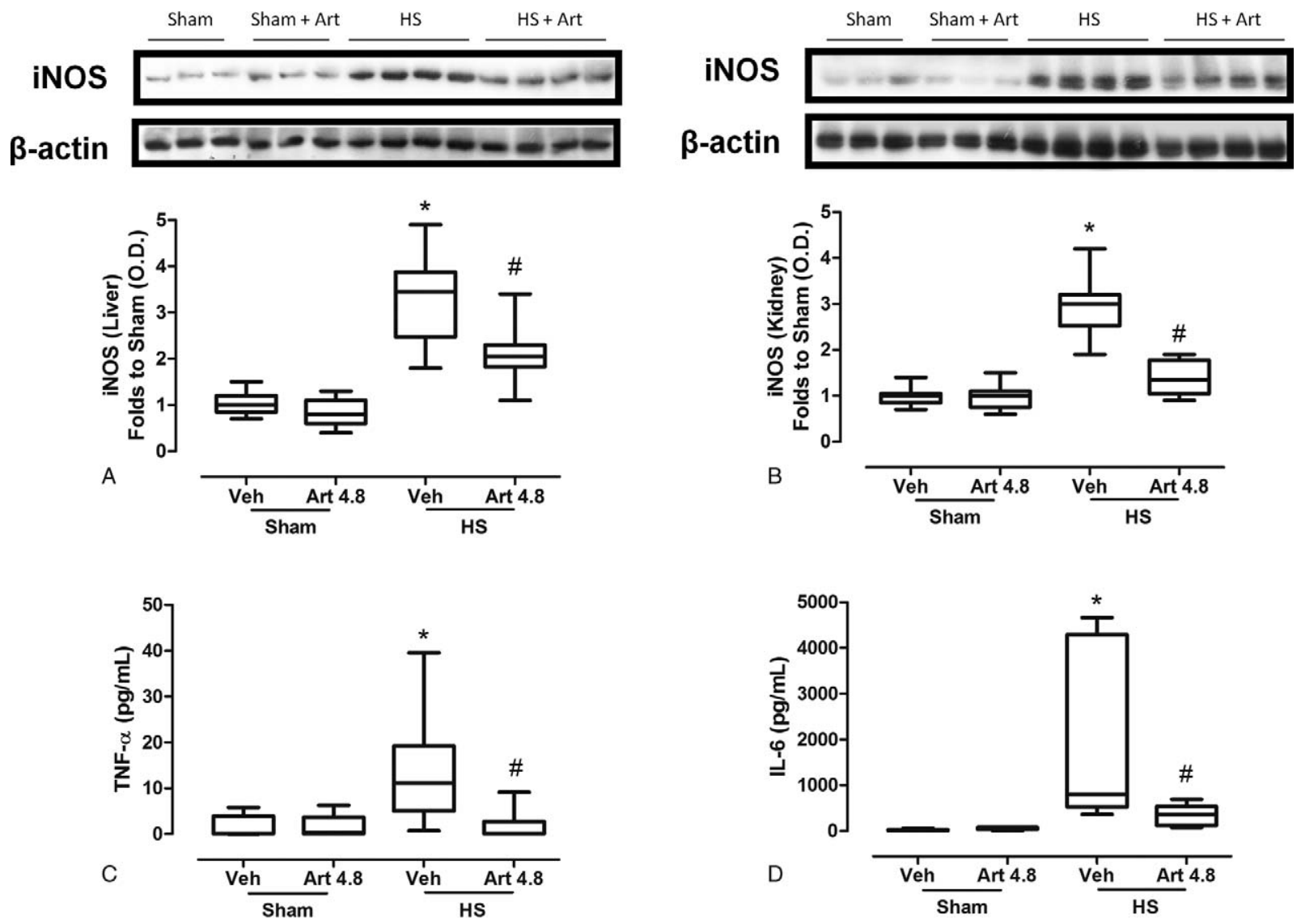

FIGURE 7. Artesunate attenuates iNOS expression in liver and kidney and serum TNF- $\alpha$ and IL-6 induced by HS. The iNOS expression in the liver $(A)$ and kidney $(B)$ of sham and HS rats treated with vehicle or artesunate $(4.8 \mathrm{mg} / \mathrm{kg})$ on resuscitation were determined by Western blotting. Protein expression was measured as relative OD, corrected for the corresponding $\beta$-actin contents and normalized using the related sham band. The serum concentrations of (C) TNF- $\alpha$ and (D) IL- 6 were determined by ELISA in sham and HS rats treated with vehicle or artesunate $(4.8 \mathrm{mg} / \mathrm{kg})$ on resuscitation. Data are presented as box and whiskers format showing medians, interquartile range, and full range $(n=8-10$ animals per group). When necessary (TNF and IL-6 data) the values had been transformed into logarithms to achieve normality and homogeneity of variances. Statistical analysis was performed using 2-way ANOVA followed by Bonferroni post hoc test. ${ }^{*} P<0.05$ vs sham + vehicle and ${ }^{\#} P<0.05$ vs HS + vehicle. ANOVA indicates analysis of variance; ELISA, enzyme-linked immunosorbent assay; OD, optical density.

attenuated iNOS expression in both the organs. As an excessive formation of NO by iNOS contributes to the organ injury in $\mathrm{HS}^{16,40}$ and sepsis, ${ }^{42,43,44}$ and also causes cellular damage and tissue injury through peroxynitrite formation, ${ }^{41}$ the reduction in iNOS expression may contribute to the organ protection afforded by artesunate. However, we have not observed a significant difference in the degree of staining for 3-NT among the groups. It is possible that iNOS expression, NO production, NT formation, and nitration of proteins occur at different stages after the onset of resuscitation. All of our data were obtained at 4 hours after HS, and we speculate that iNOS expression precedes any significant nitration of tyrosine residues within proteins. It should be stressed that our data also demonstrate that the protection afforded by artesunate is not secondary to a reduction of peroxynitrite formation.

An increase circulating proinflammatory cytokines such as TNF- $\alpha$ and IL- 6 is associated with organ injury/dysfunction and lethality after HS and sepsis. ${ }^{43,45,46,47}$ Elevated IL- 6 concentrations predict the development of MOF in patients with trauma-hemorrhage. ${ }^{48}$ We report here that HS results in the excessive formation of
TNF- $\alpha$ and IL- 6 , and that treatment of HS rats with artesunate reduced the production of these cytokines. As patients who either produce excessive amounts of cytokines or cannot clear them from the circulation have an increased risk of MOF and death ${ }^{45}$; the reduction of circulating TNF- $\alpha$ and IL- 6 may have importantly contributed to the attenuation of organ injury and dysfunction observed in HS rats treated with artesunate.

Taken together, our results show that the protective effect of artesunate in HS is associated with an increased activation of Akt and eNOS phosphorylation and consequently inhibition of GSK-3 $\beta$, which in turn, inhibited the translocation of NF- $\kappa B$ to the nucleus. This mechanism is supported on several levels. At a transcriptome level using pathway analysis, we find strong support for AKT activation on the basis of genome-wide enrichment of differential expression among AKT interactors. Expression of proinflammatory, inhibition targets of AKT, such as IRAK1 and FOXO4, are reduced. At the protein level, artesunate also attenuated iNOS expression and proinflammatory cytokines formation, all of which may have contributed for the attenuation of MOF associated with HS. Thus, the use 
of the antimalarial drug artesunate, which is a safe, low-cost and already-developed drug, could represent a therapeutic approach for the treatment and/or prevention of the MOF associated with HS.

Limitations of our study: We used an acute model of HS, which leads to MOF and systemic inflammation within a few hours of the onset of resuscitation. At this time point ( $4 \mathrm{~h}$ after resuscitation) there is organ dysfunction, significant activation of the NF-кB pathway and expression of $\mathrm{NF}-\mathrm{KB}$-dependent proteins. However, although effective in this acute setting, we cannot conclude that artesunate will have the same beneficial effect in animal models with long-term follow-up. Future studies in large animals (as pigs) may be useful to gain a better understanding of both efficacy and mechanism of action of artesunate in HS. Also, the demonstration of a reduction in mortality by means of such studies would have strengthened the efficacy data with artesunate reported in our study. We report that the administration of artesunate during resuscitation had no effect on blood pressure indicating that the beneficial effects of artesunate in HS may be independent of an increase in blood pressure/increase in perfusion of vital organs. This may limit the effectiveness of artesunate in HS, as other interventions may be necessary to overcome the vascular decompensation associated with HS.

Currently, there is no specific treatment for organ failure/ dysfunction associated with HS, which is associated with a high morbidity and mortality. Thus, a safe and low-cost therapeutic intervention may have a great impact on the patient quality of life and the costs of health care. The doses of artesunate used here are very similar to those used in patients with malaria. Artesunate has a very good safety profile with no or minimal adverse effects in both healthy volunteers (up to $8 \mathrm{mg} / \mathrm{kg}$ i.v.) and patients with severe malaria. The excellent safety profile of artesunate is (at least in part) due to its very short half-life in man: the intravenous injection of artesunate results in high, transient peak concentrations, which decline rapidly with an estimated half-life of 15 minutes. The concentration of the active metabolite DHA peaks within 25 minutes of injection of artesunate, and the half-life is estimated 30 to 60 minutes. The metabolism of artesunate is not affected by the presence of either renal or liver failure, and the dose of artesunate does not need to be adjusted in patients with multiple organ failure. ${ }^{49}$ Thus, we are planning a phase II placebo-controlled randomized clinical trial designed to evaluate the effects of GMP-artesunate $(2.4$ or $4.8 \mathrm{mg} / \mathrm{kg}$ i.v.) in 105 patients with trauma and severe hemorrhage. This singlecenter trial will commence at the Royal London Hospital in 2016.

\section{ACKNOWLEDGMENTS}

Mrs. Kristie Wood (Genome Centre at QMUL) is gratefully acknowledged for her work on RNA sequencing.

\section{REFERENCES}

1. Pfeifer R, Tarkin IS, Rocos B, et al. Patterns of mortality and causes of death in polytrauma patients — has anything changed? Injury. 2009;40:907-911.

2. Nast-Kolb D, Aufmkolk M, Rucholtz S, et al. Multiple organ failure still a major cause of morbidity but not mortality in blunt multiple trauma. J Trauma. 2001;51:835-841.

3. Minei JP, Cuschieri J, Sperry J, et al. The changing pattern and implications of multiple organ failure after blunt injury with hemorrhagic shock. Crit Care Med. 2012;40:1129-1135.

4. Jarrar D, Chaudry IH, Wang P. Organ dysfunction following hemorrhage and sepsis: mechanisms and therapeutic approaches. Int J Mol Med. 1999;4:575583.

5. WHO. Guidelines for the Treatment of Malaria. 2nd ed, Geneva, Roll Back Malaria Dept: World Health Organization; 2010.

6. Sinclair D, Donegan S, Isba R, et al. Artesunate versus quinine for treating severe malaria. Cochrane Database Syst Rev. 2012;6. CD005967.

7. Krishna S, Bustamante L, Haynes RK, et al. Artemisinins: their growing importance in medicine. Trends Pharmacol Sci. 2008;29:520-527.
8. Ho WE, Peh HY, Chan TK, et al. Artemisinins: pharmacological actions beyond anti-malarial. Pharmacol Ther. 2014;142:126-139.

9. Singh NP, Lai H. Selective toxicity of dihydroartemisinin and holotransferrin toward human breast cancer cells. Life Sci. 2001;70:49-56.

10. Efferth T, Romero MR, Wolf DG, et al. The antiviral activities of artemisinin and artesunate. Clin Infect Dis. 2008;47:804-811.

11. Moore CM, Hoey EM, Trudgett A, et al. Artemisinins act through at least two targets in a yeast model. FEMS Yeast Res. 2011;11:233-237.

12. Ho WE, Cheng C, Peh HY, et al. Anti-malarial drug artesunate ameliorates oxidative lung damage in experimental allergic asthma. Free Radic Biol Med. 2012;53:498-507.

13. Zhao M, Xue DB, Zheng B, et al. Induction of apoptosis by artemisinin relieving the severity of inflammation in caerulein-induced acute pancreatitis. World J Gastroenterol. 2007;13:5612-5617.

14. Li Y, Wang S, Wang Y, et al. Inhibitory effect of the antimalarial agent artesunate on collagen-induced arthritis in rats through nuclear factor kappa B and mitogen-activated protein kinase signaling pathway. Transl Res. 2013;161:89-98.

15. Li B, Yu M, Pan X, et al. Artesunate reduces serum lipopolysaccharide in cecal ligation/puncture mice via enhanced LPS internalization by macrophages through increased mRNA expression of scavenger receptors. Int J Mol Sci. 2014;15:1143-1161.

16. Sordi R, Chiazza F, Patel NSA, et al. Preconditioning with low dose lipopolysaccharide aggravates the organ injury/dysfunction caused by hemorrhagic shock in rats. PLoS One. 2015;10:e0122096.

17. Chen EY, Tan CM, Kou Y, et al. Enrichr: interactive and collaborative HTML5 gene list enrichment analysis tool. BMC Bioinformatics. 2013;14:128.

18. Krämer A, Green J, Pollard J Jr, et al. Causal analysis approaches in Ingenuity Pathway Analysis. Bioinformatics. 2014;30:523-530.

19. Takeda K, Akira S. TLR signaling pathways. Semin Immunol. 2004;16: 329.

20. Chassin C, Hempel C, Stockinger S, et al. MicroRNA-146a- mediated downregulation of IRAK1 protects mouse and human small intestine against ischemia/reperfusion injury. EMBO Mol Med. 2012;4:1308-1319.

21. Li H, Liang J, Castrillon DH, et al. FoxO4 regulates tumor necrosis factor alpha-directed smooth muscle cell migration by activating matrix metalloproteinase 9 gene transcription. Mol Cell Biol. 2007;27:2676-2686.

22. Sorokin AV, Chen J. MEMO1 a new IRS1-interacting protein, induces epithelial-mesenchymal transition in mammary epithelial cells. Oncogene. 2013;32:3130-3138.

23. Cantley LC. The phosphoinositide 3-kinase pathway. Science. 2002;296: $1655-1657$

24. Shu YS, Tao W, Miao QB, et al. Improvement of ventilation-induced lung injury in a rodent model by inhibition of inhibitory $\kappa \mathrm{B}$ kinase. J Trauma Acute Care Surg. 2014;76:1417-1424.

25. Khan AI, Coldewey SM, Patel NS, et al. Erythropoietin attenuates cardiac dysfunction in experimental sepsis in mice via activation of the $\beta$-common receptor. Dis Model Mech. 2013;6:1021-1030.

26. Coldewey SM, Rogazzo M, Collino M, et al. Inhibition of ІкB kinase reduces the multiple organ dysfunction caused by sepsis in the mouse. Dis Model Mech. 2013;6:1031-1042.

27. Cai Z, Semenza GL. Phosphatidylinositol-3-kinase signaling is required for erythropoietin-mediated acute protection against myocardial ischemia/reperfusion injury. Circulation. 2004;109:2050-2053.

28. Sordi R, Chiazza F, Johnson FL, et al. Inhibition of IкB kinase attenuates the organ injury and dysfunction associated with hemorrhagic shock. Mol Med. 2015;21:563-575.

29. Dimmeler S, Fleming I, Fisslthaler B, et al. Activation of nitric oxide synthase in endothelial cells by Akt-dependent phosphorylation. Nature. 1999;399:601-605.

30. Cabrales P, Tsai AG, Intaglietta M. Exogenous nitric oxide induces protection during hemorrhagic shock. Resuscitation. 2009;80:707-712.

31. Nandra KK, Collino M, Rogazzo M, et al. Pharmacological preconditioning with erythropoietin attenuates the organ injury and dysfunction induced in a rat model of hemorrhagic shock. Dis Model Mech. 2013;6:701-709.

32. Martin M, Rehani K, Jope RS, et al. Toll-like receptor-mediated cytokine production is differentially regulated by glycogen synthase kinase 3 . Nat Immunol. 2005;6:777-784.

33. Whittle BJ, Varga C, Pósa A, et al. Reduction of experimental colitis in the rat by inhibitors of glycogen synthase kinase-3beta. $\mathrm{Br} J$ Pharmacol. 2006; $147: 575-582$

34. Dugo L, Collin M, Allen DA, et al. GSK-3beta inhibitors attenuate the organ injury/dysfunction caused by endotoxemia in the rat. Crit Care Med. 2005;33:1903-1912. 
35. Dugo L, Collin M, Allen DA, et al. Insulin reduces the multiple organ injury and dysfunction caused by coadministration of lipopolysaccharide and peptidoglycan independently of blood glucose: role of glycogen synthase kinase3beta inhibition. Crit Care Med. 2006;34:1489-1496.

36. Dugo L, Abdelrahman M, Murch O, et al. Glycogen synthase kinase-3beta inhibitors protect against the organ injury and dysfunction caused by hemorrhage and resuscitation. Shock. 2006;25:485-491.

37. Schwabe RF, Brenner DA. Role of glycogen synthase kinase-3 in TNF-alphainduced NF-kappaB activation and apoptosis in hepatocytes. Am J Physiol Gastrointest Liver Physiol. 2002;283:G204-G211.

38. Senftleben U, Karin M. The IKK/NF-kappa B pathway. Crit Care Med. 2002;30:S18-26.

39. Gao C, Xu DQ, Gao CJ, et al. An exogenous hydrogen sulphide donor, NaHS inhibits the nuclear factor (B inhibitor kinase/nuclear factor (b inhibitor) nuclear factor-(B signaling pathway and exerts cardioprotective effects in a rat hemorrhagic shock model. Biol Pharm Bull. 2012;35:1029-1034.

40. Thiemermann C, Szabó C, Mitchell JA, et al. Vascular hyporeactivity to vasoconstrictor agents and hemodynamic decompensation in hemorrhagic shock is mediated by nitric oxide. Proc Natl Acad Sci U S A. 1993;90:267-271.

41. McDonald MC, Izumi M, Cuzzocrea S, et al. A novel, potent and selective inhibitor of the activity of inducible nitric oxide synthase (GW274150) reduces the organ injury in hemorrhagic shock. J Physiol Pharmacol. 2002;53:555-569.
42. Shirhan M, Moochhala SM, Kerwin SY, et al. Influence of selective nitric oxide synthetase inhibitor for treatment of refractory haemorrhagic shock. Resuscitation. 2004;61:221-229.

43. Sordi R, Fernandes D, Heckert BT, et al. Early potassium channel blockade improves sepsis-induced organ damage and cardiovascular dysfunction. $\mathrm{BrJ}$ Pharmacol. 2011;163:1289-1301.

44. Jiang $\mathrm{H}$, Huang $\mathrm{Y}, \mathrm{Xu} \mathrm{H}$, et al. Inhibition of hypoxia inducible factor-1( ameliorates lung injury induced by trauma and hemorrhagic shock in rats. Acta Pharmacol Sin. 2012;33:635-643.

45. Pinsky MR, Vincent JL, Deviere J, et al. Serum cytokine levels in human septic shock. Relation to multiple-system organ failure and mortality. Chest. 1993;103:565-575

46. Bahrami S, Yao YM, Leichtfried G, et al. Significance of TNF in hemorrhagerelated hemodynamic alterations, organ injury, and mortality in rats. Am J Physiol. 1997;272:H2219-2226.

47. Sordi R, Menezes-de-Lima O, Della-Justina AM, et al. Pneumonia-induced sepsis in mice: temporal study of inflammatory and cardiovascular parameters. Int J Exp Pathol. 2013;94:144-155.

48. Lausevic Z, Lausevic M, Trbojevic-Stankovic J, et al. Predicting multiple organ failure in patients with severe trauma. Can J Surg. 2008;51:97-102.

49. Rosenthal PJ. Artesunate for the treatment of severe falciparum malaria. $N$ Engl J Med. 2008;358:1829-1836. 